\title{
Semi-Jailing Technique Using a Neuroform3 Stent for Coiling of Wide-Necked Intracranial Aneurysms
}

\author{
Jun Kyeung Ko, M.D., Ph.D., Won Ho Cho, M.D., Ph.D., ${ }^{1}$ Seung Heon Cha, M.D., Ph.D., ${ }^{1}$ Chang Hwa Choi, M.D., Ph.D., \\ Sang Weon Lee, M.D., Ph.D., ${ }^{2}$ Tae Hong Lee, M.D., Ph.D. ${ }^{3}$ \\ Department of Neurosurgery, Diagnostic Radiology, ${ }^{3}$ Medical Research Institute, Pusan National University Hospital, Busan, Korea \\ Department of Neurosurgery, ${ }^{2}$ Medical Research Institute, Pusan National University Yangsan Hospital, Yangsan, Korea
}

Objective : The semi-jailing technique (SJT) provides stent-assisted remodeling of the aneurysm neck during coil embolization without grasping the coil delivery microcatheter. We retrospectively evaluated the efficacy and safety of SJT using a Neuroform3 stent for coiling of wide-necked intracranial aneurysms.

Methods : We collected the clinical and radiological data between January 2009 and June 2015 of the wide-necked aneurysms treated with SJT using a Neuroform3 stent.

Results : SJT using a Neuroform3 stent was attempted in 70 wide-necked aneurysms (68 patients). There were 56 unruptured and 14 ruptured aneurysms. The size of aneurysm ranged from 1.7 to $28.1 \mathrm{~mm}$ (mean $6.1 \mathrm{~mm}$ ). The immediate angiographic results were complete occlusion in 55 aneurysms (78.6\%), neck remnant in $7(10.0 \%)$, and aneurysm remnant in 8 (11.4\%). Overall, periprocedural complications occurred in 13 patients (19.1\%), including asymptomatic thromboembolism in 7 (10.3\%), symptomatic thromboembolism in 4 (5.9\%), and symptomatic hemorrhagic complications in 2 (2.9\%). Conventional angiography follow-up was obtained in 55 (78.6\%) of 70 aneurysms (mean, 10.9 months). The result showed progressive occlusion in 7 aneurysms (12.7\%) and recanalization in 1 aneurysm (1.8\%). At the end of the observation period (mean, 17.5 months), all 54 patients without subarachnoid hemorrhage showed excellent clinical outcomes (modified Rankin Scale [mRS] 0), except two (mRS 1 or 2) and seven of 14 patients with subarachnoid hemorrhage remained symptom-free (mRS 0).

Conclusion : In this report of 70 aneurysms, SJT using a Neuroform3 stent for coiling of wide-necked intracranial aneurysms showed good technical safety, as well as favorable clinical and angiographic outcomes.

Key Words : Aneurysm · Coil · Stent.

\section{INTRODUCTION}

When stent-assisted coiling (SAC) is performed for the treatment of intracranial aneurysm, the technique of catheter tip placement into the aneurysm sac can be divided into 2 methods : the catheter jailing technique and through the stent' $\mathrm{s}$ strut technique ${ }^{3,17)}$. Although the conventional catheter jailing technique eliminates some of the drawbacks of through the stent's strut technique, it does not offer a possibility of modifying the microcatheter position within the aneurysm

- Received : July 7, 2016 •Revised : November 10, 2016 •Accepted : November 26, 2016

- Address for reprints : Tae Hong Lee, M.D., Ph.D.

Department of Diagnostic Radiology, Pusan National University Hospital, 179 Gudeok-ro, Seo-gu, Busan 49241, Korea

Tel : +82-51-240-7354, Fax : +82-51-244-7534, E-mail : drcell06193@nate.com

This is an Open Access article distributed under the terms of the Creative Commons Attribution Non-Commercial License (http://creativecommons.org/licenses/by-nc/4.0) which permits unrestricted non-commercial use, distribution, and reproduction in any medium, provided the original work is properly cited. 
sac, which further limits the operator's ability to reposition the coil delivery catheter tip. As a novel method for overcoming such drawbacks of catheter jailing technique, the semijailing technique (STT), which was first described for cerebral aneurysms by Hong and colleagues in 2009, provides stent-assisted remodeling of the aneurysm neck during coil embolization without grasping the coil delivery microcatheter ${ }^{14)}$. Recently, most operators seem to choose a retrievable stent for SJT due to the ease of use with this stent ${ }^{4,11,14)}$. However, we prefer the use of an open-cell stent, especially the Neuroform stent (Boston Scientific, Fremont, CA, USA) in SAC, including SJT.

In this report, we describe SJT that had been used successfully to occlude wide-necked aneurysms with a Neuroform3 Stent Delivery System (previous version). The purpose of this study is to assess retrospectively both the efficacy and safety of SJT using a Neuroform 3 stent and to report our experiences.

\section{MATERIALS AND METHODS}

We performed a review of the clinical and radiological records of all participants during a 72-month period from January 2009 to June 2015 in a prospective single-center registry of patients that had underwent SJT using a Neuroform3 stent for coiling of wide-necked intracranial aneurysms. SJT using a Neuroform stent has been attempted in almost all cases that required SAC. Patients harboring intracranial aneurysms, ruptured or unruptured, with a wide-neck $(\geq 4 \mathrm{~mm})$ or an unfavorable dome-to-neck ratio $(<2)$ were included. Dissecting or fusiform aneurysms, blood blister-like aneurysms, aneurysms associated with a brain arteriovenous malformation, and traumatic or mycotic aneurysms were excluded. Medical data were collected prospectively in accordance to our protocol and reviewed retrospectively with an approval by the institutional review board.

\section{General information of endovascular treatment}

In general, endovascular treatment was administered as soon as possible after subarachnoid hemorrhage (SAH), regardless of the clinical condition of the patient. All patients without SAH received a dual antiplatelet medication including $75 \mathrm{mg}$ of clopidogrel and $100 \mathrm{mg}$ of acetylsalicylic acid (ASA) each day, for over five days or a loading dose of $300 \mathrm{mg}$ of clopidogrel and $300 \mathrm{mg}$ of ASA at least 12 hours before the procedure. For patients presented with $\mathrm{SAH}$, a dual antiplatelet premedication was not prescribed due to the risk of rebleeding. All procedures were performed via the unilateral or bilateral transfemoral approach under intravenous sedation with full anticoagulation via the use of intravenous heparin. The activated clotting time was maintained at 2-3 times the baseline throughout the procedure. The appropriate Neuroform 3 stent size was selected (available diameters were 3.0-4.5 $\mathrm{mm}$ and lengths were $15-30 \mathrm{~mm}$ ) such that the stent diameter was $0.5 \mathrm{~mm}$ larger than the parent vessel diameter and the ends of the device would extend at least $5 \mathrm{~mm}$ beyond the neck of the aneurysm on each side.

\section{Semi-jailing technique}

The femoral artery is first accessed using a 6 Fr $80 \mathrm{~cm}$ long Shuttle sheath (Cook, Bloomington, IN, USA) and a 6 F Envoy guiding catheter (Cordis, Miami Lakes, FL, USA) is navigated into the ipsilateral petrous segment of internal carotid artery for anterior circulation aneurysms or into the distal second segment of the vertebral artery for posterior circulation aneurysms. A microcatheter for coil delivery is placed into the aneurysm sac using a guidewire, and one or two loops of the first coil are deployed. Pre-deployed coil loops can be used as a guidewire and may allow re-introduction of the microcatheter tip into the sac when the catheter tip is extruded from the aneurysm sac during stent deployment. Additional microcatheter application for two-catheter technique, which usually requires bilateral transfemoral approach, was optional. A Neuroform 3 stent is then delivered using a $300 \mathrm{~cm}$ long exchange microwire over the portion of aneurysm, and partially deployed approximately one-third to reduce the effective neck size and to prevent distal coil migration. Because the coil delivery catheter is not jailed to the vessel wall, it remains freely maneuverable during the procedure and permit the passive kick-back movement of the microcatheter while decreasing the risk of aneurysm perforation during coil packing. The stent is fully deployed after achieving the dense coil packing. Alternatively, in the event that the coil packing density is unsatisfactory on a control angiography, coiling can continue using the jailed microcatheter. Another advantage is to fix the distal portion of the final coil that had difficulty being inserted further, between the stent and the vessel wall. The procedure is illustrated in Fig. 1. 

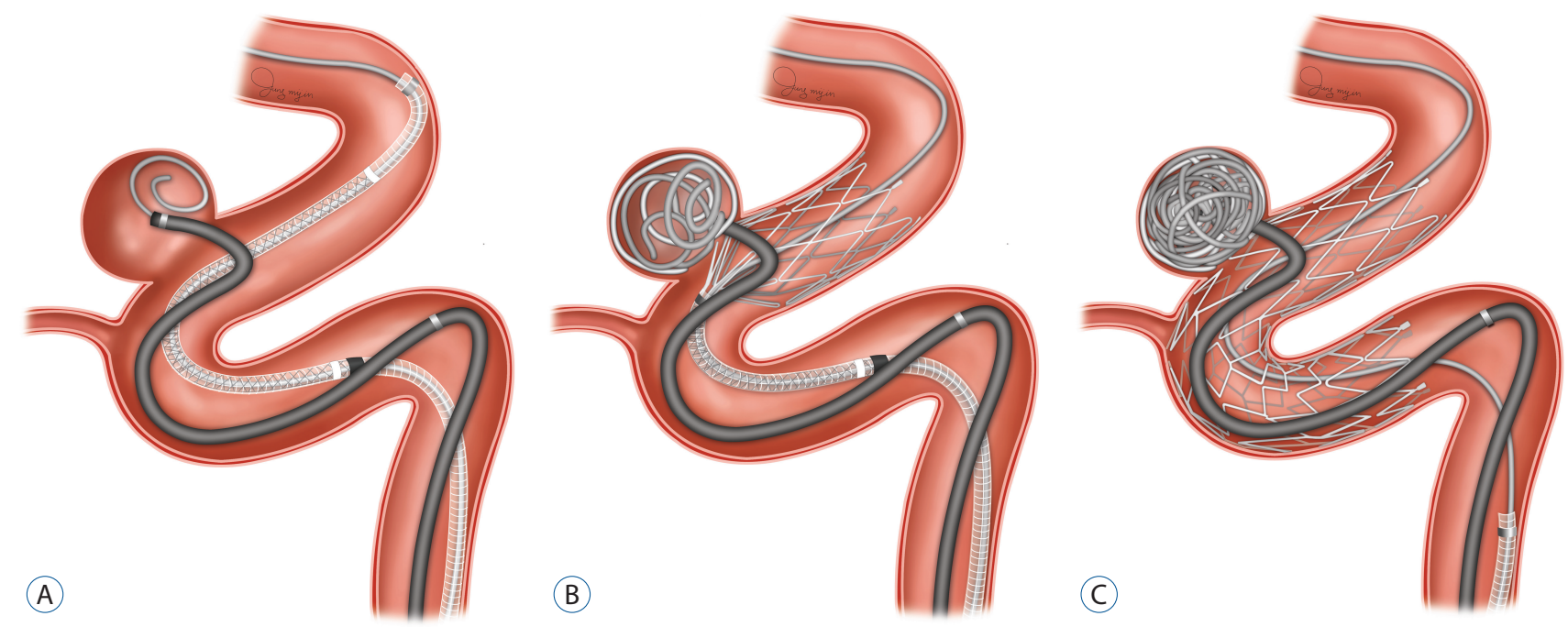

Fig. 1. Schematic illustration of semi-jailing technique using a Neuroform3 stent. A : After selection of the aneurysm with microcatheter with partially deployed coil, the Neuroform3 stent is then delivered over the aneurysm portion. B : After the stent is partially deployed to cover the part of aneurysm neck, homogeneous coil framing is achieved without coil protrusion by the limitation of the partially-deployed stent. Because the coil delivery catheter is not jailed to the vessel wall, it moves freely within the sac and provides better control over the coil mass. C: The stent is fully deployed after achieving the dense coil packing, or coiling can be continued using the jailed microcatheter.

\section{After endovascular treatment}

Immediately after the procedure, all patients underwent a complete neurological examination performed by a vascular neurosurgeon, and non-enhanced brain computed tomography scan to evaluate for possible hemorrhagic complications. All patients received subcutaneous administration of $2850 \mathrm{IU}$ of low-molecular heparin (Fraxiparin, GlaxoSmithKline, Marly-le-Roi Cedex, France) twice or three times per day, for a duration of five days. After the procedure, all patients with SAH were prescribed with dual antiplatelet medication, which consisted of a loading dose of $300 \mathrm{mg}$ of clopidogrel and 300 mg of ASA. In all patients, dual antiplatelet drugs, which consisted of clopidogrel (75 mg) and ASA (100 mg) each day were also maintained for at least six months. Clopidogrel resistance test was performed approximately 24 hours after drug administration according to our protocol of SAC in all cases. In patients were poor responders to clopidogrel, signaled by VerifyNow P2Y12 assay, cilostazol was added.

\section{Analysis}

Immediate and follow-up angiographic results were categorized into three groups based on the Raymond classification ${ }^{22}$. Recanalization and progressive occlusion were defined as a change in the Raymond classification, a comparison of the rate of occlusion postembolization and on follow-up. Recanalization was defined as worsening of classification : complete occlusion to neck remnant, neck remnant to aneurysm remnant, or complete occlusion to aneurysm remnant. Progressive occlusion was defined as the improvement of classification. A significant in-stent stenosis was defined as greater than $50 \%$ narrowing or complete occlusion of the parent vessel at the time of follow-up. Radiologic assessments were conducted by two independent blinded neuroradiologists. Clinical results were assessed upon discharge from the hospital, or at the last clinical visit, using the modified Rankin Scale (mRS). Fisher exact tests were used to compare proportions. A probability value of $<0.05$ was considered statistically significant.

\section{RESULTS}

\section{Patient population and aneurysm morphology}

Patient demographic data and characteristics of aneurysms are provided in Table 1. SJT using a Neuroform3 stent was attempted in 70 wide-necked aneurysms (68 patients). There were 54 females and 14 males with a mean age of 58.0 years (range, 38 to 82 years). Fifty-six aneurysms (80.0\%) were unruptured and $14(20.0 \%)$ were ruptured. The sizes of an aneu- 
rysm and neck ranged from 1.7 to $28.1 \mathrm{~mm}$ (mean $6.1 \mathrm{~mm}$ ) and from 1.9 to $10.3 \mathrm{~mm}$ (mean $4.0 \mathrm{~mm}$ ), respectively. The average dome-to-neck ratio was 1.2. Most aneurysms (88.6\%) were located in the anterior circulation, and the carotid-ophthalmic segment was the most frequent location $(44.3 \%)$. Thirteen cases (18.6\%) were treated with SJT as a salvaging technique after the failure of other neck remodeling techniques (e.g. two-catheters or balloon); the 57 remaining aneurysms $(81.4 \%)$ were treated primarily with SJT.

Table 1. Patient demographic data and characteristics of the aneurysm

\begin{tabular}{|c|c|}
\hline Number of patients treated & 68 \\
\hline Number of aneurysms treated & 70 \\
\hline Mean age, y (SD) & $58.0( \pm 7.1)$ \\
\hline \multicolumn{2}{|l|}{ Sex distribution } \\
\hline Male & $14(20.6 \%)$ \\
\hline Female & $54(79.4 \%)$ \\
\hline Mean maximum diameter of aneurysm, mm (SD) & $6.1( \pm 3.7)$ \\
\hline \multicolumn{2}{|l|}{ Aneurysm size } \\
\hline$<10 \mathrm{~mm}$ & $65(92.9 \%)$ \\
\hline $10-25 \mathrm{~mm}$ & $4(5.7 \%)$ \\
\hline$>25 \mathrm{~mm}$ & $1(1.4 \%)$ \\
\hline Mean size of aneurysm neck, mm (SD) & $4.0( \pm 1.5)$ \\
\hline Mean dome-to-neck ratio (SD) & $1.2( \pm 0.5)$ \\
\hline Anterior circulation & $62(88.6 \%)$ \\
\hline ICA-Cavernous segment & 1 \\
\hline ICA-Clinoid segment & 8 \\
\hline ICA -Ophthalimic segment & 31 \\
\hline ICA -Communicating segment & 17 \\
\hline ACOM & 3 \\
\hline Pericallosal artery & 1 \\
\hline MCA & 1 \\
\hline Posterior circulation & $8(11.4 \%)$ \\
\hline Basilar artery & 6 \\
\hline Vertebral artery & 1 \\
\hline PCA & 1 \\
\hline Ruptured aneurysm & $14(20.0 \%)$ \\
\hline Unruptured aneurysm & $56(80.0 \%)$ \\
\hline
\end{tabular}

ACA : anterior cerebral artery, ICA : internal carotid artery, MCA : middle cerebral artery, PCA : posterior cerebral artery, SD : standard deviation

\section{Immediate angiographic results and initial pro- cedural complications}

Procedural complications and results of treatment are shown in Table 2. SJT was successful in all cases. The stent was deployed to the intended location, and the wide neck of aneurysm was accurately and completely covered in all patients, except in two cases. Stent malpositioning secondary to inadvertent liberation occurred in two cases (2.9\%), which was associated with a very tortuous internal carotid artery, making the navigation with the delivery system difficult, and once the inadvertent deployment was detected, we could not retrieve the stent, turning full deployment at the petrous segment the only option. A second Neuroform3 stent was placed through the stent, over the neck of aneurysm without any major trouble. Four aneurysms were treated with a double stent placement (stent-within-stent) in order to solve the problem of coil protrusion through the struts. Immediate post-procedural angiograms showed complete occlusion in 55 aneurysms (78.6\%), neck remnant in 7 aneurysms (10.0\%), and aneurysm

Table 2. Procedural complications and treatment results

\begin{tabular}{lc}
\hline Stent strategy, N (\%) & $57(81.4)$ \\
Planned & $13(18.6)$ \\
Salvaging & \\
Symptomatic complications, $\mathrm{n}(\%)$ & $4(5.9)$ \\
Thromboembolism & $2(2.9)$ \\
Intraprocedural rupture & \\
Procedure-related, N (\%) & $1(1.4)$ \\
Mortality & $5(7.1)$ \\
Morbidity & \\
Immediate angiographic results, N (\%) & $55(78.6)$ \\
Complete & $7(10.0)$ \\
Near complete & $8(11.4)$ \\
Partial & \\
Follow-up angiographic results, N (\%) & $7(12.7)$ \\
Progressive occlusion & $1(1.8)$ \\
Recanalization & $1(1.8)$ \\
In-stent stenosis & \\
mRS, n (\%) & $63(92.6)$ \\
0-2 & $5(7.4)$ \\
\hline 3-6 & \\
\hline
\end{tabular}

$\bar{N}$ : number of aneurysms treated, $\mathrm{n}$ : number of patients treated, $\mathrm{mRS}$ : modified Rankin Score 
remnant in 8 aneurysms (11.4\%).

Overall, periprocedural complications occurred in 13 patients (19.1\%) : asymptomatic thromboembolic complications in 7 (10.3\%), symptomatic thromboembolic complications in 4 (5.9\%), and symptomatic hemorrhagic complications in 2 (2.9\%). All hemorrhagic complications were symptomatic. Therefore, 6 (8.8\%) out of the 68 patients experienced symptomatic periprocedural complications in this study. Eleven patients with thromboembolic complication underwent intraarterial thrombolysis; Chemical and/or mechanical thrombolysis, using intra-arterial infusion of abciximab (Reopro ${ }^{\circledR}$; a potent inhibitor of hemostasis) or balloon, was attempted in all cases. Seven of these patients experienced successful recanalization without any symptoms (Fig. 2). However, one patient had a permanent visual deficit due to occlusion of the ophthalmic artery. Three patients showed a cerebral infarct in a variety of sizes in postoperative magnetic resonance imaging. Nonetheless, these patients were significantly improved by 6 months to an $\mathrm{mRS}$ of 0 or 1 . Among these 11 patients, there were no hemorrhagic complications related to using abciximab. Hemorrhagic complications, on the other hand, occurred in 2 patients $(2.9 \%)$ and all were symptomatic. One patient underwent intraprocedural rupture of the initially unruptured aneurysm; Endosaccular coil packing was continued, and, fortunately, no negative long-term effects were observed. The other patient with a ruptured aneurysm of carotid-communicating segment (Hunt and Hess grade III) died of massive extravasation from the aneurysm, which was the result of coil perforation during the procedure. Thus, the periprocedural mortality in our cohort was $1.4 \%$ (one of 70 procedures) and morbidity was $7.1 \%$ (five of 70 procedures).

\section{Follow-up angiographic results}

Conventional angiography follow-up was obtained in 55 (78.6\%) out of 70 aneurysms at intervals ranging from two to 24 months (mean, 10.9 months). Of the 55 aneurysms, recanalization was observed in 1 aneurysm (1.8\%) and progressive occlusion was observed in 7 aneurysms (12.7\%) (Fig. 3). A complete occlusion was obtained in 51 (92.7\%) out of 55 aneurysms at a follow-up compared with 55 (78.6\%) out of 70 aneurysms in a postprocedure evaluation. The number of aneurysms with a residual neck remnant decreased from 7 aneurysms $(10.0 \%)$ to $1(1.8 \%)$. The number of aneurysms with a residual aneurysm remnant decreased from 8 (11.4\%) to 3 aneurysms (5.5\%) (Table 3). None of the patients underwent additional coiling during the follow-up period. All parent and branch arteries with stenting were patent with no evidence of significant in-stent stenosis, except in one case (1.8\%). This patient had asymptomatic severe in-stent stenosis that was discovered 9 months later from a routine follow-up angiography. Additional balloon angioplasty of the stenotic segment was performed.

\section{Delayed complications and clinical results}

At the end of the observation period, with a mean of 17.5 months (range, 1-53 months), all 54 patients without SAH had excellent clinical outcomes (mRS 0); however, two patients were discharged with an mRS score of 1 or 2 (resulting from thromboembolic complications). In 14 patients with SAH, seven remained symptom-free (mRS 0); while the other seven had various disabilities by the end of the observation period, including two with an mRS score of 6 (resulting from
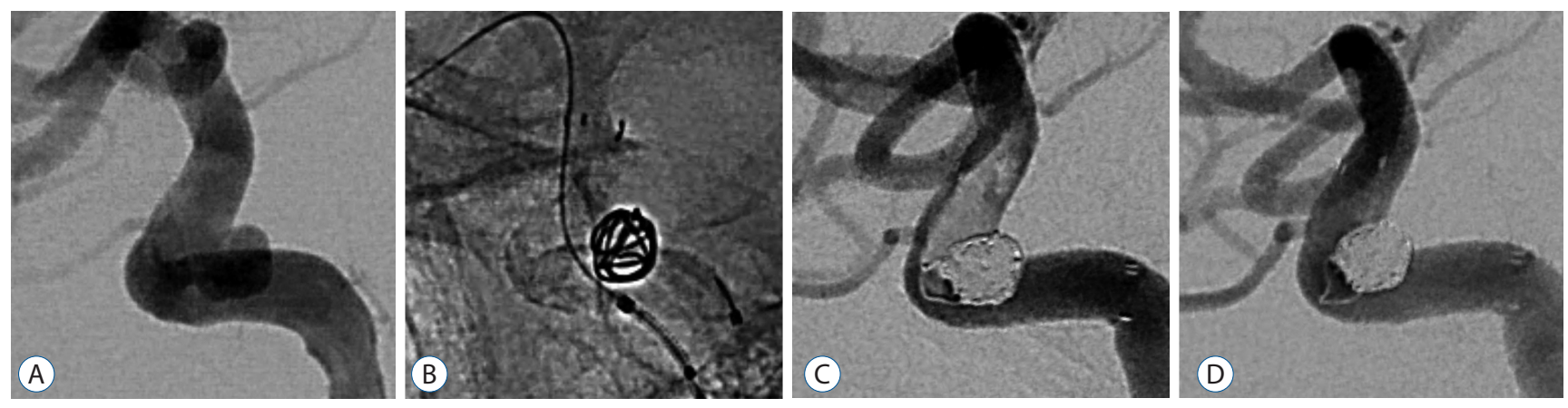

Fig. 2. A : A 63-year-old female with an unruptured aneurysm of the left carotid-ophthalmic segment. B : After the first coil is delivered into the aneurysm, a stent is partially deployed across the aneurysm neck. C : Immediate post-operative angiogram shows a definite filling defect along the stent wall representing acute in-stent thrombosis. D : After intra-arterial administration of $10 \mathrm{mg}$ abciximab, acute thrombosis completely disappeared on the angiogram performed 15 minutes later. 

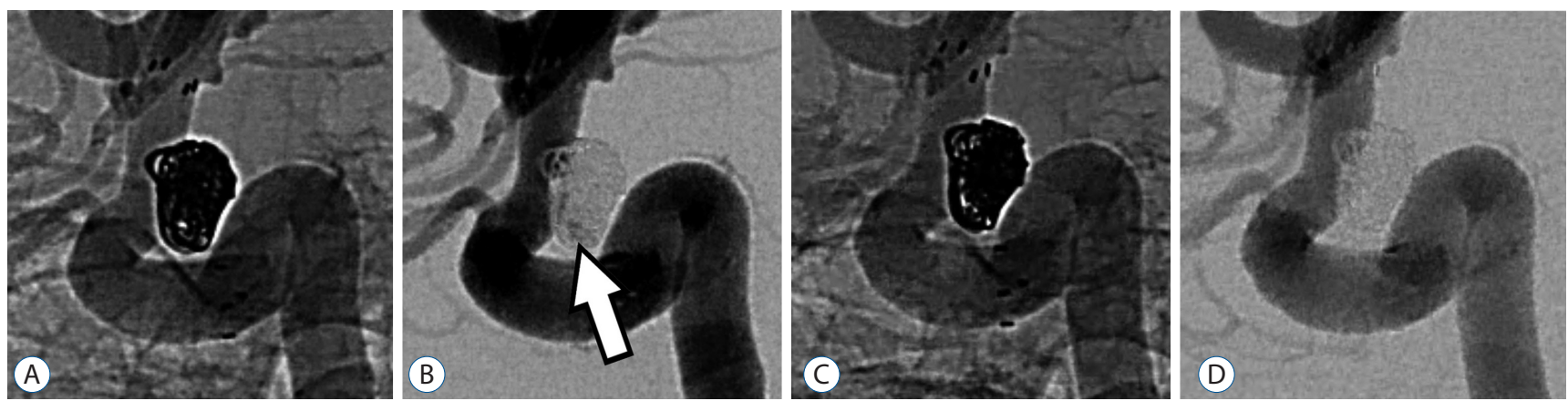

Fig. 3. A 54-year-old female with a wide-necked aneurysm arising from carotid-ophthalmic segment. A and B : Unsubtracted and subtracted images acquired immediately after SJT-assisted coiling show residual aneurysm filling (arrow). C and D : The 12-month follow-up angiograms reveal a completely occluded aneurysm, which may be attributed to coil remodeling and progressive thrombosis, and patent parent artery. SJT : semi-jailing technique.

Table 3. Comparison between results of postprocedural occlusion and occlusion at follow-up

\begin{tabular}{lcc}
\hline & Postprocedure $(\mathbf{n}=\mathbf{7 0})$ & Follow-up $(\mathbf{n}=\mathbf{5 5})$ \\
\hline Complete occlusion & $55(78.6)$ & $51(92.7)$ \\
Neck remnant & $7(10.0)$ & $1(1.8)$ \\
Aneurysm remnant & $8(11.4)$ & $3(5.5)$ \\
\hline
\end{tabular}

Values are presented as $n(\%) . n$ : number of aneurysms treated

hemorrhagic complications or poor preoperative status), three with an mRS score of 5 (resulting from poor preoperative status) and two with an mRS score of 2 (resulting from poor preoperative status). During the observation period, with a mean of 17.5 months (range, 1-53 months), no delayed thromboembolic or hemorrhagic complication was observed in any of the surviving patients.

\section{DISCUSSION}

In our experience, the conventional catheter jailing technique has several advantages over through the strut technique. First, the through the stent's strut technique has been reported to sometimes have difficulties accessing the aneurysm for coiling after the initial stent placement, especially when the parent artery is tortuous, when the aneurysm is small, or when the closed and small-sized cell design stents, such as an Enterprise (Cordis Endovascular, Miami Lakes, FL, USA), is used. Furthermore, if the microcatheter is forced out of the aneurysm during coiling, re-entry through the stent struts may also be challenging and hazardous. Second, like a balloon, the deployed stent prevents the kick-back movement of the jailed catheter during coiling. Third, coil herniation into the parent artery is less likely to occur in the catheter jailing technique. However, the catheter jailing technique also has some drawbacks. First, the catheter tip is extruded from the aneurysm sac during stent deployment. One solution is to deploy several coil loops before stent placement. Pre-deployed coil loops can be used as a guidewire, which may allow reintroduction of the microcatheter tip into the sac. Second, it does not offer the possibility of modifying the microcatheter position within the aneurysm sac, further limiting the operator's ability to appropriately address complex aneurysms. Third, it does not permit a passive kick-back movement of the microcatheter during dense coil packing, which result in a higher risk of aneurysm rupture by coil perforation. As a novel method for overcoming such drawbacks of catheter jailing technique, SJT was first reported by Hong et al. in $2009^{14}$. SJT provides a stent-assisted remodeling of the aneurysm neck during coil embolization without grasping the coil delivery microcatheter. They described several advantages of SJT, compared with the previous techniques : STT provides all the benefits of SAC while maintaining a higher degree of microcatheter flexibility and maneuverability during coil deployment as with balloon-assisted coiling. Unlike previous techniques, which resulted in fixed and complete coverage of the aneurysm neck, SJT provides a variable control over the effective aneurysm neck size and working space for the microcatheter. Furthermore, the effective size of aneurysm neck can be altered depending on other factors, such as the selected coil size 
and the position of the coil mass. SJT gives the practitioner a much broader range of working positions, facilitating a compartmental approach. This can be critical to matching the coil mass geometry with that of the lesion, enabling an optimum packing while retaining the control needed to maintain patency of important arteries that may originate from the aneurysm. They used only a retrievable stent (Enterprise), whereas, in our series, the non-retrievable stent with over-the-wire delivery system (Neuroform 3 stent) was only used. Since a stent is partially deployed in front of the aneurysm neck to act as a remodeling device in SJT, most operators choose a retrievable device such as Solitaire AB or Enterprise for SJT ${ }^{4,11,14)}$. These retrievable stents present several advantages : the possibility to regain access to the aneurysm sac in case of kick-back by a slight repositioning of the stent; the possibility to choose to either retrieve or definitely deploy the stent after coiling; and the possibility of not using a double antiplatelet treatment if the stent is retrieved at the end of the procedure.

We prefer the use of an open-cell stent, especially the Neuroform 3 Stent Delivery System (previous version), in SAC including SJT for the following reasons : Placement of the overlapping stents or ballooning within a stent requires fewer steps because the microwire is left in place for loading subsequent stents or balloons, providing a key advantage over the Neuroform 3 design. In addition, this microwire can be used as a guidewire, which may allow quickly access to additional devices when the distal complication occurs. In our series, technical success was achieved in all 70 cases with $1.4 \%$ periprocedural mortality (one of 70 procedures) and $7.1 \%$ periprocedural morbidity (five of 70 procedures), and $88.6 \%$ complete occlusion or neck remnant. Hong et al. ${ }^{14)}$ reported their experience of SJT using only a retrievable stent. They described that technical success was achieved in all 22 cases with $4.5 \%$ periprocedural mortality (one of 22 procedures) and no periprocedural morbidity, and $91 \%$ complete occlusion or neck remnant. A comparison between Hong et al. ${ }^{14)}$ 's and our series demonstrates the incidences of complete occlusion and periprocedural complication are not statistically significant $(p>0.05)$. This finding may advocate for broader stent options for the SJT.

However, the delivery and deployment of Neuroform 3 stent across the sharp angles formed by parent vessels are also very troublesome. In this situation, we place a guiding catheter further distal into the internal carotid artery or the vertebral artery and a rigid 0.014-in microwire (Transend 14; Target/Bos- ton Scientific, Natick, MA, USA) is also navigated as far as possible. These techniques allowed for successful tracking of the Neuroform3 stent and implanting into target vessels. However, although navigation of the stent delivery system to the targeted landing zone is not a limitation, the actual deployment of the stent is frequently difficult because of binding of the microwire, the stabilizer, and the stent delivery catheter. The tortuosity of the carotid siphon often creates numerous points of friction within open-cell designed stent delivery system, and these technical difficulties with deployment result in inadvertent deployment and malposition of stents ${ }^{9}$. Beyond that, the high porosity and the design of the struts make the Neuroform stent system highly flexible, but are large enough to allow protrusion and migration of coils ${ }^{2,15}$.

We think that SJT might have a slightly higher risk of thromboembolic complications than conventional SAC (catheter jailing or through the stent's strut technique). Because the SJT design, which involves the simultaneous presence of 2 catheters and partially deployed stent in the parent artery, may limit flow or increase the risk of thromboembolic event. Prior series of SAC have indicated thromboembolism rates approximating $4-8 \%{ }^{7,10)}$. Conversely, we experienced a relatively high rate of acute thrombosis after SJT in 11 cases $(16.2 \%$, nine in unruptured aneurysms and two in SAH patients). However, symptomatic thromboembolism occurred in 4 cases (5.9\%), similar with previous reports of conventional SAC. In a direct comparison of complication rates between Enterprise and Neuroform stents, Kadkhodayan et al. and Durst et al. both reported increased risk of thromboembolic complications with Enterprise stents ${ }^{6,16}$. The open cell design of the Neuroform stent allows it to conform to the curvature of the native vessel with good wall apposition ${ }^{8,18}$. Conversely, the Enterprise stent uses a closed cell design, which has been demonstrated to result in ovalization and kinking or buckling. This design can result in poor apposition to the vessel wall ${ }^{8,12)}$. In a study evaluating MR angiographic outcomes following Enterprise and Neuroform stent placement, a semilunar signal pattern along the outside of the stent signaled poor wall apposition. This finding, termed the crescent sign, was identified in only the Enterprise cohort and was correlated with ipsilateral DWI signal abnormalities ${ }^{13)}$. Narrowing and kinking are likely to alter the flow dynamics, whereas poor wall apposition can lead to blood stagnation, providing an impetus for thrombus formation. When the closed and small-sized cell design stents, 
such as the Enterprise, is used in SJT, stent cell size of the deployed part becomes smaller, as compared with an open cell stent. These additional intraluminal "hardware" is thought to cause stagnant flow and increase the likelihood of thromboembolic event. This is also the main reason why we stick to the Neuroform stent for the SJT. Even though Solitaire AB has the largest cell size among stents for intracranial use, the microwire is not easy to pass through its struts due to its overlap design, especially in the tortuous segment of intracranial artery such as a carotid siphon, based on our experience. Therefore, when a stent thrombosis occurs in SAC using a Solitaire AB, it is often faced with difficult situation.

However, SAC requires dual antiplatelet therapy to minimize the thromboembolic risk related to stent placement ${ }^{19,23)}$. In the setting of SAH, these drugs significantly heighten the risk of hemorrhagic complications related to ventriculostomy placement, aneurysmal rebleeding, and possible need for future invasive procedures ${ }^{5)}$. Nonetheless, several studies have reported safe, successful treatment of ruptured aneurysms with SAC despite these worries ${ }^{1,20,21,23)}$. In our 14 patients with a ruptured aneurysm, SJT was performed without a dual antiplatelet premedication. Even though one case of intraprocedural rupture occurred as the result of coil perforation, no patients had clinically significant intracranial hemorrhagic complications after the procedure.

\section{CONCLUSION}

In this retrospective report, successful coil embolization of wide-necked aneurysms was achieved using SJT with a Neuroform 3 stent in 70 consecutive cases. The proposed SJT-assisted coiling is a feasible treatment option for reconstruction of wide-necked aneurysms. Further studies with larger patient series and a longer follow up period will be helpful in elucidation of both the efficacy and the longevity of this treatment.

\section{References}

1. Amenta PS, Dalyai RT, Kung D, Toporowski A, Chandela S, Hasan D, et al. : Stent-assisted coiling of wide-necked aneurysms in the setting of acute subarachnoid hemorrhage : experience in 65 patients. Neurosurgery 70 : 1415-1429; discussion 1429, 2012
2. Benitez RP, Silva MT, Klem J, Veznedaroglu E, Rosenwasser RH : Endovascular occlusion of wide-necked aneurysms with a new intracranial microstent (Neuroform) and detachable coils. Neurosurgery 54 : 1359-1367; discussion 1368, 2004

3. Biondi A, Janardhan V, Katz JM, Salvaggio K, Riina HA, Gobin YP : Neuroform stent-assisted coil embolization of wide-neck intracranial aneurysms : strategies in stent deployment and midterm follow-up. Neurosurgery 61 : 460-468; discussion 468-469, 2007

4. Byun JS, Kim JK, Lee HY, Hwang SN : Temporary semi-jailing technique for coil embolization of wide-neck aneurysm with small caliber parent artery following incomplete clipping. J Korean Neurosurg Soc 53 : 241-244, 2013

5. Chung J, Lim YC, Suh SH, Shim YS, Kim YB, Joo JY, et al. : Stent-assisted coil embolization of ruptured wide-necked aneurysms in the acute period : incidence of and risk factors for periprocedural complications. J Neurosurg $121:$ 4-11, 2014

6. Durst CR, Khan P, Gaughen J, Patrie J, Starke RM, Conant P, et al. : Direct comparison of Neuroform and Enterprise stents in the treatment of wide-necked intracranial aneurysms. Clin Radiol 69 : e471-e476, 2014

7. Fargen $\mathrm{KM}$, Hoh BL, Welch BG, Pride GL, Lanzino G, Boulos AS, et al. : Long-term results of enterprise stent-assisted coiling of cerebral aneurysms. Neurosurgery 71 : 239-244; discussion 244, 2012

8. Fernandez Zubillaga A, Guglielmi G, Vinuela F, Duckwiler GR : Endovascular occlusion of intracranial aneurysms with electrically detachable coils : correlation of aneurysm neck size and treatment results. AJNR Am J Neuroradiol 15 : 815-820, 1994

9. Fiorella D, Albuquerque FC, Han P, McDougall CG : Preliminary experience using the Neuroform stent for the treatment of cerebral aneurysms. Neurosurgery 54 : 6-16; discussion 16-17, 2004

10. Fiorella D, Albuquerque FC, Woo H, Rasmussen PA, Masaryk TJ, MCDougall CG : Neuroform stent assisted aneurysm treatment : evolving treatment strategies, complications and results of long term follow-up. J Neurointerv Surg 2 : 16-22, 2010

11. Gao X, Liang G, Li Z, Qu H, Wei X : Stent-assisted coil embolization of wide-necked intracranial aneurysms using a semi-deployment technique : angiographic and clinical outcomes in 31 consecutive patients. Interv Neuroradiol $16: 385-393,2010$

12. Heller RS, Malek AM : Parent vessel size and curvature strongly influence risk of incomplete stent apposition in enterprise intracranial aneurysm stent coiling. AJNR Am J Neuroradiol 32 : 1714-1720, 2011

13. Heller RS, Miele WR, Do-Dai DD, Malek AM : Crescent sign on magnetic resonance angiography revealing incomplete stent apposition : correlation with diffusion-weighted changes in stent-mediated coil embolization of aneurysms. J Neurosurg 115 : 624-632, 2011

14. Hong B, Patel NV, Gounis MJ, DeLeo MJ 3rd, Linfante I, Wojak JC, et al. : Semi-jailing technique for coil embolization of complex, wide-necked intracranial aneurysms. Neurosurgery 65 : 1131-1138; discussion 11381139, 2009

15. Howington JU, Hanel RA, Harrigan MR, Levy El, Guterman LR, Hopkins $L N$ : The Neuroform stent, the first microcatheter-delivered stent for use in the intracranial circulation. Neurosurgery $54: 2-5,2004$ 
16. Kadkhodayan Y, Rhodes N, Blackburn S, Derdeyn CP, Cross DT 3rd, Moran CJ : Comparison of Enterprise with Neuroform stent-assisted coiling of intracranial aneurysms. AJR Am J Roentgenol 200 : 872-878, 2013

17. Kim BM, Kim DJ, Kim DI : Stent application for the treatment of cerebral aneurysms. Neurointervention $6:$ 53-70, 2011

18. Krischek O, Miloslavski E, Fischer S, Shrivastava S, Henkes H : A comparison of functional and physical properties of self-expanding intracranial stents [Neuroform3, Wingspan, Solitaire, Leo+, Enterprise]. Minim Invasive Neurosurg $54: 21-28,2011$

19. Lopes D, Sani $S$ : Histological postmortem study of an internal carotid artery aneurysm treated with the Neuroform stent. Neurosurgery 56 : E416; discussion E416, 2005

20. Meckel S, Singh TP, Undren P, Ramgren B, Nilsson OG, Phatouros C, et al. : Endovascular treatment using predominantly stent-assisted coil embolization and antiplatelet and anticoagulation management of ruptured blood blister-like aneurysms. AJNR Am J Neuroradiol 32 : 764-771, 2011

21. Mocco J, Snyder KV, Albuquerque FC, Bendok BR, Alan SB, Carpenter $J S$, et al. : Treatment of intracranial aneurysms with the Enterprise stent : a multicenter registry. J Neurosurg $110:$ 35-39, 2009

22. Raymond J, Guilbert F, Weill A, Georganos SA, Juravsky L, Lambert A, et al. : Long-term angiographic recurrences after selective endovascular treatment of aneurysms with detachable coils. Stroke 34 : 1398-1403, 2003

23. Tahtinen OI, Vanninen RL, Manninen HI, Rautio R, Haapanen A, Niskakangas $T$, et al. : Wide-necked intracranial aneurysms : treatment with stent-assisted coil embolization during acute ( $<72$ hours) subarachnoid hemorrhage--experience in 61 consecutive patients. Radiology 253 : 199-208, 2009 